\title{
Determinantes de las horas de trabajo de las mujeres en México: un enfoque de pseudopanel (2005-2010)
}

\author{
Armando Sánchez V., Verónica Villarespe R., \\ Diego A. Román C. y Ana Liz Herrera M.
}

\section{Resumen}

Las horas de trabajo de las mujeres mexicanas no solo dependen de los salarios y las características individuales, sino también de factores relacionados con la estructura del hogar, que generan incentivos para que las mujeres limiten sus horas de trabajo remunerado. En este estudio se utiliza un pseudopanel con cinco millones de observaciones de la Encuesta Nacional de Ocupación y Empleo relativo al período 2005-2010. Se analizan cohortes de edad de la población femenina trabajadora y un modelo de pseudopanel que mide la sensibilidad de las horas laborales de las mujeres ante variaciones en el salario y factores relacionados con la estructura del hogar, como la disponibilidad de ayuda en casa y la presencia de niños. Se encontró que las horas trabajadas por las mujeres aumentan cuando en el hogar vive otra mujer adulta, mientras que la presencia de niños y de otro adulto varón las afecta negativamente.

Palabras clave

Mujeres, empleo de la mujer, horas de trabajo, medición, modelos econométricos, análisis por cohortes, México

Clasificación JEL

J21, J12, J16, C33

Autores

Armando Sánchez Vargas es Investigador Titular de tiempo completo nivel "C" del Departamento de Coordinación de Análisis Macroeconométrico Prospectivo del Instituto de Investigaciones Económicas de la Universidad Nacional Autónoma de México (UNAM). armando_sanchez123@hotmail.com

Verónica Villarespe Reyes es Investigadora Titular de tiempo completo nivel "C" del Departamento de Historia Económica del Instituto de Investigaciones Económicas de la Universidad Nacional Autónoma de México (UNAM). reyesvv@unam.mx

Diego Alí Román Cedillo es Licenciado en Economía de la Universidad Nacional Autónoma de México (UNAM). droman87@hotmail.com

Ana Liz Herrera Merino es Profesora Adjunta de Asignatura nivel "B" de la Facultad de Economía de la Universidad Nacional Autónoma de México (UNAM). anita_hm90@hotmail.com 


\section{Introducción}

De acuerdo con cifras de la Organización para la Cooperación y el Desarrollo Económicos (OCDE), el ritmo de incorporación de las mujeres al mercado laboral en México ha sido muy lento en los últimos años, pues su participación pasó del 36,67\% en 2005 al 38,32\% en 2012. En la literatura empírica sobre diversos países, esta tendencia se ha atribuido frecuentemente a la dinámica de los salarios y a características individuales. Sin embargo, no se han analizado en profundidad los efectos que podrían tener otros factores de tipo cultural, social y demográfico en las horas de trabajo remunerado de las mujeres ${ }^{1}$. La hipótesis central de este estudio consiste en que, además de los factores de carácter económico, la estructura de los hogares también podría condicionar la participación laboral femenina, ya que puede generar incentivos para que las mujeres reduzcan su participación en el mercado laboral. Esto se debe a que las mujeres no solo eligen cuántas horas destinarán a un trabajo remunerado, sino también la cantidad de tiempo que dedicarán a la producción de bienes dentro del hogar y al cuidado de los integrantes de la familia, papeles tradicionalmente asignados a las mujeres y que compiten con su uso laboral del tiempo (Acosta, Perticará y Ramos, 2006). Por ejemplo, la presencia de varios niños pequeños en la composición del hogar podría generar incentivos para que la mujer abandone el trabajo remunerado y dedique su tiempo al cuidado, debido a los roles culturales y sociales de la mujer, la falta de ayuda dentro del hogar y la escasez de servicios de cuidado públicos. En este contexto, surgiría la necesidad de formular e implementar políticas públicas orientadas a liberar una parte del tiempo que las mujeres emplean en actividades domésticas y de cuidado en el hogar, para que puedan dedicarla a un trabajo remunerado si así lo desean. En general, se trata de impulsar políticas que contribuyan a disminuir las barreras de tipo social, cultural o demográfico que podrían limitar las horas de trabajo y las oportunidades de progreso de las mujeres.

La población objetivo de este estudio son las mujeres de 12 a 65 años de edad. Para los cálculos sobre los determinantes de las horas trabajadas se utilizó la técnica econométrica de pseudopanel, con un enfoque de cohortes por años de nacimiento. La base de datos empleada incluye alrededor de 5,2 millones de observaciones y contiene información de la Encuesta Nacional de Ocupación y Empleo (ENOE) que abarca desde el tercer trimestre de 2005 al segundo trimestre de 2010 (INEGl, s/f). Asimismo, se determinaron las elasticidades mediante diferentes métodos de estimación para la muestra con la finalidad de asegurar la robustez estadística de los resultados. No se encontró en la literatura un análisis de los efectos de la composición del hogar en las horas de trabajo de las mujeres que se base en una muestra tan extensa y aumente la confiabilidad de las estimaciones de sus determinantes utilizando métodos econométricos de pseudopanel para México, tal como se hace en este estudio (Deaton, 1997).

Los resultados sugieren que la estructura del hogar incide en las horas que las mujeres ofrecen en el mercado laboral. Por una parte, la disponibilidad de tiempo para trabajar aumenta cuando en el hogar hay otra mujer mayor de 14 años, que podría representar una ayuda no remunerada en el quehacer doméstico y el cuidado del hogar y se traduciría en un incremento del número de horas para ofrecer en dicho mercado. Por otra, la presencia de niños tiene un efecto negativo en las horas laborales de las mujeres, pues su cuidado puede requerir una mayor demanda de tiempo (ArceoGómez y Campos-Vázquez, 2010). En consecuencia, la estructura del hogar es un factor relevante en la determinación y la disponibilidad de horas para trabajar de las mujeres. Esto podría explicarse porque las necesidades de los integrantes del hogar condicionan las decisiones de las mujeres en el ámbito laboral.

El artículo se divide en cinco secciones, incluida esta Introducción. En la segunda se efectúa una revisión de la literatura existente y se advierte que en investigaciones previas se ha sugerido que

A lo largo del texto se utiliza "horas trabajadas" para hacer referencia a las horas de trabajo remunerado de las mujeres. 
la estructura del hogar podría incidir en las horas de trabajo de las mujeres. En la tercera sección se presenta evidencia empírica a nivel de cohorte sobre el número medio de horas por semana que las mujeres en México destinan a trabajar. Asimismo, se muestra la variación que sufren dichas horas cuando se condicionan a aspectos relacionados con la estructura del hogar. En la cuarta sección se muestran los resultados obtenidos a partir del modelo de pseudopanel y con diferentes estimadores, que garantizan la robustez de los resultados, y en la quinta y última se realizan algunos comentarios finales.

\section{Evidencia previa sobre la participación laboral femenina y su relación con la estructura del hogar}

El análisis de los factores que determinan la incorporación de las mujeres al mercado de trabajo ha cobrado mucha importancia en los últimos años. Domínguez y Brown (2013) estudian en profundidad el papel que desempeñan las diferencias de género en la participación laboral en México. Entre las conclusiones más relevantes de dicho estudio destaca que la presencia de hijos y adultos mayores en el hogar podría afectar la decisión de la mujer de trabajar en forma remunerada en el domicilio o fuera de él. Las autoras también sugieren que el trabajo pagado desde el domicilio es funcional en México porque las labores de cuidado, tradicionalmente asignadas a la mujer, podrían dificultar la participación laboral femenina en el mercado formal. Sin embargo, la decisión de llevar a cabo trabajo remunerado en el hogar también podría reflejar la insuficiencia del número de escuelas y guarderías con horarios adecuados para que las madres tengan más opciones de trabajo formal remunerado. En ese sentido, en un estudio realizado por Arceo-Gómez y Campos-Vázquez (2010) se muestra que las horas de trabajo pagado de las mujeres mexicanas que tienen niños menores de cinco años son mucho más sensibles a las variaciones en el salario que las de una mujer mexicana promedio. Esto podría atribuirse a que los roles femeninos tradicionales dentro del hogar limitan a las mujeres en la elección de la distribución de su tiempo.

Attanasio, Low y Sánchez (2008) estudian los determinantes de las variaciones en la oferta laboral de las mujeres con hijos en tres diferentes cohortes en los Estados Unidos, sobre la base de un modelo de ciclo de vida. Los resultados de su investigación muestran que tanto la disminución de los costos monetarios del cuidado de los niños como el aumento de los salarios incentivan de manera positiva la participación femenina en el mercado laboral. Por otra parte, Ludin, Mörk y Öckert (2008) estiman los efectos de la reducción de los costos del cuidado de los niños en la oferta laboral femenina en los Estados Unidos y sugieren que dicho impacto es significativo pero heterogéneo, pues depende del tipo de familia y de la región objeto de estudio. Warunsiri y McNown (2010) estiman los determinantes de la oferta de trabajo femenina en Tailandia a partir de la construcción y el análisis de diferentes cohortes sintéticas de mujeres. El principal hallazgo de esos autores es que existe una relación negativa entre el salario y las horas de trabajo para las mujeres de ese país (la elasticidad salario de la oferta laboral fue de alrededor de -0,25). En este artículo se argumenta que la existencia de una curva de oferta de trabajo con pendiente negativa se podría relacionar con los usos competitivos del tiempo de una mujer (Dessing, 2002). Es decir, un aumento en los salarios podría disminuir las horas trabajadas de las mujeres, ya que estas podrían optar por dedicar más tiempo a actividades como el cuidado de los hijos y la prestación de servicios de uso doméstico². Otra conclusión relevante del mismo estudio es que, si bien las horas trabajadas de las mujeres solteras son más sensibles a una

\footnotetext{
2 Para aquellos que están trabajando, un cambio en el salario induce tanto efectos ingreso como efectos sustitución, con consecuencias opuestas en lo que se refiere a las horas de trabajo. Si bien la expectativa normal es que los efectos ingresos superen a los efectos sustitución, de manera que la elasticidad de las horas trabajadas con respecto a los salarios sea positiva, la evidencia de varios países en desarrollo rechaza dicha suposición.
} 
modificación salarial en comparación con las casadas, su probabilidad de trabajar es menor que la de estas últimas. En este contexto, en otras investigaciones como las de Schultz (1990), Dessign (2002) y Warunsiri y McNown (2010) se ha concluido que en países como Tailandia, el Perú y Filipinas también existe una elasticidad negativa del salario por horas trabajadas.

En otros trabajos sobre el tema referidos a países en desarrollo, y en los que se aplican técnicas de pseudopanel, como el de Bassi (2003), se concluye que pese a que las elasticidades salario de las horas trabajadas son positivas al igual que en los países desarrollados, la diferencia radica en que su magnitud es sustancialmente pequeña. Así, se puede anticipar que la cantidad de horas de trabajo de las mujeres en dichos países podría explicarse por otras variables, que posiblemente se relacionen con el uso del tiempo y las labores tradicionales de las mujeres en el hogar, como el cuidado de los hijos y tareas domésticas como lavar, planchar y cocinar. También es frecuente que uno de los principales motivos por el cual las mujeres se incorporan al mercado laboral sea mantener un determinado nivel de ingresos en el hogar, tal como señala Licona (2000) en su estudio sobre el efecto de la pobreza en el suministro de mano de obra femenina en México. Esto se ha confirmado en otros estudios, incluido el de Dasgupta y Goldar (2005) relativo a las mujeres indígenas en condiciones de pobreza. Así, es posible concluir que los determinantes de la oferta laboral femenina podrían estar condicionados por factores muy diferentes con respecto a los de los hombres (Juhn y Murphy, 1996).

Por último, es importante mencionar que existen pocos estudios empíricos que hagan hincapié en la incidencia de la estructura del hogar en las horas de trabajo de las mujeres y que, específicamente, analicen si la presencia de otros adultos que podrían realizar actividades domésticas no remuneradas o niños en el hogar representarían una ayuda o una barrera a la incorporación de las mujeres al mercado laboral. Con la presente investigación se busca cubrir ese vacío en la literatura tradicional y ofrecer evidencia empírica al respecto mediante técnicas de pseudopanel con un enfoque de cohortes aplicadas a la relación entre la estructura del hogar y las horas laborales de las mujeres en México.

\section{Marco teórico}

El fundamento teórico del análisis de este artículo es el modelo de ciclo de vida de cohortes de la oferta laboral femenina de Attanasio, Low y Sánchez (2008). En dicho modelo, los hogares enfrentan incertidumbre acerca de los salarios de los hombres y las mujeres, la maternidad es exógena y los niños imponen un costo monetario fijo cuando las madres deciden trabajar. Básicamente, el modelo explica los cambios en la oferta laboral femenina y asume que los hogares maximizan su utilidad de esperanza de vida. La función de utilidad que se desarrolla es intertemporalmente separable y la utilidad instantánea depende del consumo por persona en el hogar y de la elección de oferta laboral de la esposa.

En particular, se considera un hogar con una función de utilidad instantánea de la forma:

$$
u_{t}=u\left(c_{t}, P_{t}, e_{t}\right)
$$

Donde $P_{t}$ es una variable discreta $\{0,1\}$ que muestra la decisión de la mujer en su oferta laboral, $c_{t}$ es el consumo total del hogar y $e_{t}$ es el número de adultos equivalentes en el hogar.

Por otra parte, dentro del mismo modelo se incluyen las unidades de cuidado de los niños que necesita una familia cuyo primer hijo tiene la edad $a_{t}$ como $G\left(a_{t}\right)$. El precio de cada unidad de cuidado se expresa como $p$. Así, el costo total del cuidado de los niños que enfrenta un hogar cuando la mujer participa en el mercado laboral está dado por:

$$
F\left(a_{t}\right)=p G\left(a_{t}\right)
$$


El estudio es relevante en términos de la elección de la mujer de incorporarse al mercado de trabajo. La información proporcionada indica que solo las mujeres más productivas permanecen en el mercado laboral después de tener hijos. Además, se puede apreciar la importancia potencial de la elección de continuar trabajando o no después de tener hijos, así como las repercusiones de esa decisión en la experiencia y el salario de las mujeres.

\section{Datos y resultados}

\section{Hechos estilizados de las horas de trabajo remunerado de las mujeres en México}

Para analizar las horas de trabajo remunerado de las mujeres en México se trabajó con los datos trimestrales de la Encuesta Nacional de Ocupación y Empleo (ENOE) relativos a los 32 estados del país. El período de análisis va desde el tercer trimestre de 2005 al segundo trimestre de 2010. La ENOE es una encuesta representativa a nivel nacional que proporciona información estadística ocupacional y sociodemográfica. Si bien el tipo de muestreo es aleatorio, las viviendas seleccionadas se sustituyen mediante un esquema rotatorio en el que la quinta parte de la muestra que ya cumplió un ciclo de cinco visitas de los encuestadores se reemplaza cada tres meses. Así, en cada trimestre se mantiene el $80 \%$ de la muestra.

El análisis estadístico se aplicó únicamente a las mujeres que cumplen con las siguientes características: i) estar empleada; ii) tener un número positivo de horas laborales durante la semana anterior, y iii) tener un ingreso mensual. Es importante mencionar que la muestra incluye empleadas de los sectores privado y público, así como trabajadoras por cuenta propia. Para ofrecer evidencia empírica de la relación que existe entre las variables relacionadas con la estructura del hogar y las horas trabajadas por las mujeres de manera temporal se realizó un análisis estadístico a nivel de cohortes. Para ello, se construyeron nueve cohortes a partir de los años de nacimiento de la población objetivo tomando en cuenta las características mencionadas previamente y que cumplieran con el requisito de estar en edad de trabajar (de 12 a 65 años de edad).

Los datos indican que el promedio de horas que las mujeres dedican por semana a trabajar ha tenido una evolución favorable a través de las cohortes (véase el cuadro 1). Se observa que las mujeres de la cohorte 1 (1940-1950) trabajan en promedio 5,46 horas menos que sus pares de la cohorte 9 (1985-1992). Además, se aprecia que las integrantes de la cohorte 8 (1980-1985) son las que dedican más horas a trabajar a la semana (40,33 horas), no obstante, su nivel salarial no es tan alto como el de la cohorte 4 (1960-1965), que en promedio trabaja 2,9 horas menos.

En el gráfico 1 se muestra el efecto del ciclo de vida en cada cohorte: se observa que el número medio de horas trabajadas por las mujeres aumenta conforme aumentan las cohortes de 1 a 3 (1965-1970). No obstante, a partir de esta última el promedio de horas laborales desciende de una generación a otra. Esto podría indicar que las mujeres de mayor edad y las más jóvenes son las que tienen un menor número de horas trabajadas. Por otra parte, el mayor número de horas se registra en la cohorte de las mujeres nacidas entre 1965 y 1970. 
Cuadro 1

México: ingreso y horas ocupadas a la semana por cohorte de mujeres empleadas

\begin{tabular}{lccc}
\hline \multicolumn{2}{l}{ Cohorte } & $\begin{array}{c}\text { Promedio de } \\
\text { horas ocupadas }\end{array}$ & $\begin{array}{c}\text { Mediana del } \\
\text { ingreso real }^{\mathrm{a}}\end{array}$ \\
\hline 1 & $1940-1950$ & 34,55 & 1718,37 \\
\hline 2 & $1950-1955$ & 36,36 & 2237,03 \\
\hline 3 & $1955-1960$ & 36,99 & 2582,30 \\
\hline 4 & $1960-1965$ & 37,40 & 2767,25 \\
\hline 5 & $1965-1970$ & 37,87 & 2724,89 \\
\hline 6 & $1970-1975$ & 38,15 & 2691,84 \\
\hline 7 & $1975-1980$ & 38,92 & 2724,89 \\
\hline 8 & $1980-1985$ & 40,33 & 2643,89 \\
\hline 9 & $1985-1992$ & 40,01 & 2148,98 \\
\hline
\end{tabular}

Fuente: Elaboración propia, sobre la base de datos de la Encuesta Nacional de Ocupación y Empleo (ENOE).

Nota: Se realizó una prueba de medias para ambos grupos (con ayuda y sin ayuda) y la diferencia en las horas trabajadas resultó estadísticamente significativa.

a El ingreso real se refiere al ingreso mensual en pesos declarado por las personas ocupadas. Para cambiarlo a valores reales se utilizó el Índice Nacional de Precios al Consumidor (INPC) de cada trimestre del período 2005-2010. Se presenta la mediana del salario, ya que debido a la distribución sesgada de los datos es la mejor medida de tendencia central.

\section{Gráfico 1}

México: efecto del ciclo de vida en las horas semanales de trabajo de las mujeres, por cohorte

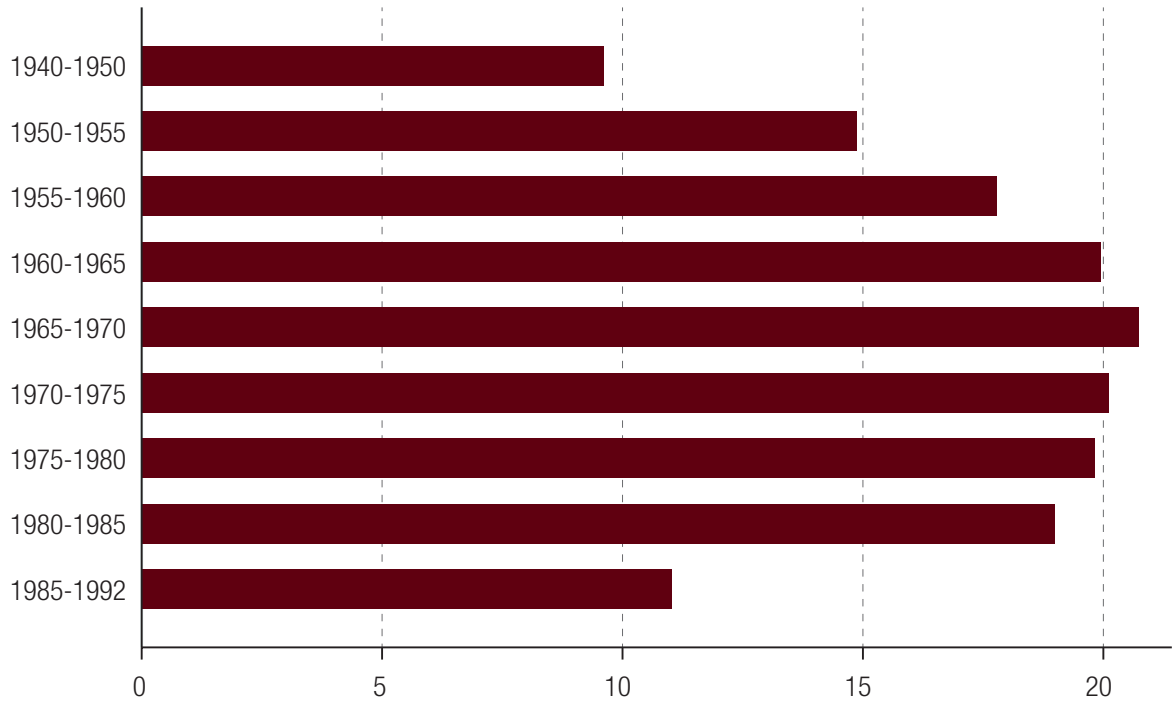

Fuente: Elaboración propia, sobre la base de datos de la Encuesta Nacional de Ocupación y Empleo (ENOE).

De los datos del cuadro 2 surge que la presencia de un adulto en el hogar, que podría representar una ayuda no remunerada para las mujeres en las labores domésticas, está asociada al incremento de sus horas de trabajo. Por ejemplo, las mujeres de la cohorte 8 (1980-1985) trabajan en promedio 4,6 horas más cuando hay otro adulto en el hogar que cuando no lo hay. En general, todas las cohortes resultan beneficiadas en cuanto a las horas de trabajo cuando hay otro adulto en el hogar. De hecho, la disponibilidad para incorporarse al mercado laboral parece incrementarse cuando existe la ayuda potencial de otra persona adulta en el hogar. Así, la presencia de otros adultos en el hogar podría incidir positivamente en las horas que las mujeres destinan al trabajo remunerado. 


\section{Cuadro 2}

México: promedio de horas trabajadas por las mujeres según la disponibilidad de ayuda de otro adulto en el hogar, por cohorte

\begin{tabular}{llcc}
\hline \multicolumn{2}{l}{ Cohorte } & Con ayuda de otro adulto & Sin ayuda de otro adulto \\
\hline 1 & $1940-1950$ & 34,99 & 33,85 \\
\hline 2 & $1950-1955$ & 36,33 & 36,44 \\
\hline 3 & $1955-1960$ & 36,96 & 37,10 \\
\hline 4 & $1960-1965$ & 37,46 & 37,19 \\
\hline 5 & $1965-1970$ & 38,20 & 37,17 \\
\hline 6 & $1970-1975$ & 39,34 & 36,66 \\
\hline 7 & $1975-1980$ & 41,02 & 36,45 \\
\hline 8 & $1980-1985$ & 41,73 & 37,12 \\
\hline 9 & $1985-1992$ & 40,46 & 37,28 \\
\hline
\end{tabular}

Fuente: Elaboración propia, sobre la base de datos de la Encuesta Nacional de Ocupación y Empleo (ENOE).

Nota: Se realizó una prueba de medias para ambos grupos (con ayuda y sin ayuda) y la diferencia en las horas trabajadas resultó estadísticamente significativa.

En el cuadro 3 se indica que de la cohorte 3 en adelante (1955-1960), la presencia de al menos un niño menor de 6 años en el hogar está asociada a un menor número de horas de trabajo de las mujeres, con respecto a los hogares que no tienen niños. Esta tendencia se mantiene en las cohortes siguientes. Es importante mencionar que el número de horas que las mujeres dejan de trabajar cuando tienen niños aumenta a través de las cohortes. Es decir, las mujeres de las cohortes 7 y 8 que tienen niños menores de 6 años trabajan 4,8 y 4,4 horas menos, respectivamente, que aquellas que no tienen niños. Esto sugiere que el cuidado de los niños podría incidir negativamente en las horas de trabajo de las mujeres en México, tal vez debido a la gran cantidad de tiempo que requiere. Se optó por estudiar el efecto de este grupo de niños debido a que en trabajos previos se ofrece evidencia de que las mujeres con hijos menores de 5 años están menos incorporadas al mercado de trabajo que el promedio de mujeres sin hijos menores de 5 años. Esta falta de asociación puede deberse a que los hijos imponen limitaciones importantes a la asignación del tiempo (Arceo-Gómez y Campos-Vázquez, 2010).

\section{Cuadro 3}

México: promedio de horas trabajadas por las mujeres empleadas según la presencia de niños menores de 6 años en el hogar, por cohorte

\begin{tabular}{lrcc}
\hline \multicolumn{2}{l}{ Cohorte } & $\begin{array}{c}\text { Tienen niños menores } \\
\text { de } 6 \text { años }\end{array}$ & $\begin{array}{c}\text { No tienen niños } \\
\text { menores de } 6 \text { años }\end{array}$ \\
\hline 1 & $1940-1950$ & 34,83 & 34,54 \\
\hline 2 & $1950-1955$ & 37,49 & 36,34 \\
\hline 3 & $1955-1960$ & 36,28 & 37,01 \\
\hline 4 & $1960-1965$ & 35,72 & 37,52 \\
\hline 5 & $1965-1970$ & 35,90 & 38,27 \\
\hline 6 & $1970-1975$ & 35,74 & 39,10 \\
\hline 7 & $1975-1980$ & 35,61 & 40,45 \\
\hline 8 & $1980-1985$ & 36,93 & 41,43 \\
\hline 9 & $1985-1992$ & 38,69 & 40,31 \\
\hline
\end{tabular}

Fuente: Elaboración propia, sobre la base de datos de la Encuesta Nacional de Ocupación y Empleo (ENOE).

Nota: La diferencia de horas de trabajo en ambos grupos para las cohortes 1, 2 y 3 es mínima debido a que el número de observaciones de las personas de dichas cohortes con niños menores de 6 años es pequeño. Se realizó una prueba de medias para ambos grupos (con niños y sin niños) y la diferencia en las horas trabajadas resultó estadísticamente significativa. 
Por otra parte, la información del cuadro 4 permite confirmar que la presencia de niños en el hogar se relaciona fuertemente con una reducción de las horas de trabajo remunerado de las mujeres. Cuando en el hogar hay niños que tienen entre 6 y 14 años de edad, el promedio de horas de trabajo de las mujeres se reduce en todas las cohortes. En este caso, los datos muestran que las mujeres de la cohorte 7 (1975-1980) son las que trabajan un menor número de horas cuando tienen niños de este rango de edad. Al contrario, las mujeres de la cohorte 9 son las que no parecen trabajar menos si tienen hijos pequeños, aunque dicha relación positiva está presente.

Cuadro 4

México: promedio de horas trabajadas por las mujeres empleadas según la presencia de niños de 6 a 14 años en el hogar, por cohorte

\begin{tabular}{lccc}
\hline \multicolumn{2}{l}{ Cohorte } & $\begin{array}{c}\text { Tienen niños de } \\
6 \text { a } 14 \text { años }\end{array}$ & $\begin{array}{c}\text { No tienen niños de } \\
6 \text { a } 14 \text { años }\end{array}$ \\
\hline 1 & $1940-1950$ & 34,83 & 34,53 \\
\hline 2 & $1950-1955$ & 35,76 & 36,44 \\
\hline 3 & $1955-1960$ & 35,88 & 37,35 \\
\hline 4 & $1960-1965$ & 36,08 & 38,34 \\
\hline 5 & $1965-1970$ & 36,56 & 39,59 \\
\hline 6 & $1970-1975$ & 36,48 & 40,30 \\
\hline 7 & $1975-1980$ & 36,45 & 40,61 \\
\hline 8 & $1980-1985$ & 39,15 & 40,78 \\
\hline 9 & $1985-1992$ & 39,90 & 40,07 \\
\hline
\end{tabular}

Fuente: Elaboración propia, sobre la base de datos de la Encuesta Nacional de Ocupación y Empleo (ENOE).

Nota: La diferencia de horas de trabajo en ambos grupos para las cohortes 1, 2 y 3 es mínima debido a que el número de observaciones de las personas de dichas cohortes con niños de 6 a 14 años es pequeño. Se realizó una prueba de medias para ambos grupos (con niños y sin niños) y la diferencia en las horas trabajadas resultó estadísticamente significativa.

Los cuadros anteriores sugieren que la presencia de un adulto adicional en el hogar se asocia de manera positiva con el número de horas que las mujeres podrían destinar al trabajo remunerado. Por otra parte, la presencia de niños en el hogar se asocia de manera inversa con el número de horas trabajadas. En la próxima sección se estiman modelos para establecer la validez estadística de esas relaciones causales. Sin embargo, se puede anticipar que es posible que dichas situaciones se deban a que los niños requieren de cuidados y atenciones que podrían afectar la disponibilidad de horas de las mujeres y hacer más costosa la decisión de trabajar en caso de no disponer de la ayuda de otro adulto en el hogar.

En el cuadro 5 se realiza el cruce de información para las mujeres que viven en hogares en los que hay al menos un adulto adicional y al menos un niño menor de 6 años. A partir de dicha intersección se puede confirmar que en la mayoría de las cohortes la presencia de otro adulto que podría realizar labores domésticas no remuneradas en el hogar permite a las mujeres destinar un mayor número de horas al trabajo remunerado a pesar de tener niños pequeños. En este caso, la mayor diferencia de horas entre las mujeres con niños menores de seis años que cuentan con ayuda y las que no se observa en la cohorte 8 (1980-1985).

Otra manera de ver la relación entre la presencia de otro adulto y las horas de las mujeres en el mercado laboral es a través de un cruce con sus niveles de educación. Del cuadro 6 surge que las mujeres con un bajo nivel educativo son las que trabajan más ante la presencia de otro adulto en el hogar. Por el contrario, las mujeres con un alto nivel educativo no parecen trabajar un mayor número de horas ante la presencia de otro adulto y niños pequeños en el hogar. Es importante destacar que las mujeres de las cohortes 7 y 8 son aquellas que, independientemente del nivel educativo, trabajan un mayor número de horas cuando tienen ayuda de otro adulto en casa. 


\section{Cuadro 5}

México: promedio de horas trabajadas por las mujeres empleadas que tienen niños menores de 6 años según la presencia de otro adulto en el hogar, por cohorte

\begin{tabular}{llcc}
\hline \multicolumn{2}{l}{ Cohorte } & $\begin{array}{c}\text { Con ayuda de otro adulto } \\
\text { niños menores de 6 años }\end{array}$ & $\begin{array}{c}\text { Sin ayuda de otro adulto y } \\
\text { niños menores de 6 años }\end{array}$ \\
\hline 1 & $1940-1950$ & 35,01 & 33,86 \\
\hline 2 & $1950-1955$ & 37,75 & 36,45 \\
\hline 3 & $1955-1960$ & 36,92 & 37,17 \\
\hline 4 & $1960-1965$ & 36,31 & 37,47 \\
\hline 5 & $1965-1970$ & 36,20 & 37,77 \\
\hline 6 & $1970-1975$ & 36,80 & 37,77 \\
\hline 7 & $1975-1980$ & 38,65 & 38,40 \\
\hline 8 & $1980-1985$ & 42,10 & 39,96 \\
\hline 9 & $1985-1992$ & 41,53 & 40,28 \\
\hline
\end{tabular}

Fuente: Elaboración propia, sobre la base de datos de la Encuesta Nacional de Ocupación y Empleo (ENOE).

Nota: Se realizó una prueba de medias para ambos grupos (con ayuda y sin ayuda) y la diferencia en las horas trabajadas resultó estadísticamente significativa.

\section{Cuadro 6}

México: ingreso y horas trabajadas por las mujeres empleadas según el nivel educativo, por cohorte

\begin{tabular}{|c|c|c|c|c|c|c|c|c|c|}
\hline \multirow{2}{*}{\multicolumn{2}{|c|}{ Cohorte }} & \multicolumn{2}{|c|}{ Preescolar-Primaria } & \multicolumn{2}{|c|}{ Secundaria-Medio superior } & \multicolumn{2}{|c|}{ Superior } & \multicolumn{2}{|c|}{ Posgrado } \\
\hline & & $\begin{array}{c}\text { Media } \\
\text { de horas } \\
\text { ocupadas }\end{array}$ & $\begin{array}{l}\text { Mediana } \\
\text { del ingreso } \\
\text { real }^{\mathrm{a}}\end{array}$ & $\begin{array}{c}\text { Media } \\
\text { de horas } \\
\text { ocupadas }\end{array}$ & $\begin{array}{l}\text { Mediana } \\
\text { del ingreso } \\
\text { real }^{\mathrm{a}}\end{array}$ & $\begin{array}{c}\text { Media } \\
\text { de horas } \\
\text { ocupadas }\end{array}$ & $\begin{array}{l}\text { Mediana } \\
\text { del ingreso } \\
\text { reala }^{\mathrm{a}}\end{array}$ & $\begin{array}{c}\text { Media } \\
\text { de horas } \\
\text { ocupadas }\end{array}$ & $\begin{array}{c}\text { Mediana } \\
\text { del ingreso } \\
\text { real }^{\mathrm{a}}\end{array}$ \\
\hline \multicolumn{10}{|c|}{ Con ayuda de otro adulto } \\
\hline 1 & $1940-1950$ & 35,07 & 1595,98 & 36,48 & 3040,62 & 35,53 & 5901,05 & 34,52 & 9116,52 \\
\hline 2 & 1950-1955 & 35,99 & 1833,22 & 37,51 & 3133,63 & 36,22 & 6347,52 & 37,13 & 12301,48 \\
\hline 3 & $1955-1960$ & 37,11 & 1917,40 & 37,63 & 3133,63 & 35,87 & 6319,28 & 37,83 & 13584,27 \\
\hline 4 & 1960-1965 & 37,38 & 1922,74 & 38,48 & 2982,71 & 35,69 & 5830,01 & 38,62 & 9349,95 \\
\hline 5 & $1965-1970$ & 37,47 & 1949,60 & 39,24 & 2706,95 & 36,51 & 5410,39 & 36,99 & 9837,72 \\
\hline 6 & $1970-1975$ & 38,01 & 1960,05 & 40,53 & 2588,95 & 38,35 & 5008,08 & 35,05 & 8232,13 \\
\hline 7 & $1975-1980$ & 40,31 & 1983,68 & 42,34 & 2566,51 & 39,49 & 4637,43 & 39,51 & 5549,22 \\
\hline 8 & $1980-1985$ & 42,75 & 2066,33 & 42,90 & 2472,20 & 39,09 & 4 099,05 & 38,26 & 5224,20 \\
\hline 9 & 1985-1992 & 41,59 & 1854,15 & 40,56 & 2133,64 & 37,50 & 3159,64 & 26,00 & 4435,16 \\
\hline \multicolumn{10}{|c|}{ Sin ayuda de otro adulto } \\
\hline 1 & $1940-1950$ & 33,87 & 1342,22 & 35,41 & 2729,43 & 34,25 & 6466,88 & 37,25 & 12041,64 \\
\hline 2 & $1950-1955$ & 35,92 & 1663,41 & 37,89 & 3122,92 & 36,62 & 6709,92 & 37,80 & 11085,10 \\
\hline 3 & $1955-1960$ & 36,83 & 1788,82 & 38,16 & 3128,35 & 36,40 & 6821,29 & 41,55 & 12485,75 \\
\hline 4 & $1960-1965$ & 37,51 & 1854,15 & 38,09 & 3090,26 & 35,78 & 6319,28 & 39,12 & 13525,97 \\
\hline 5 & $1965-1970$ & 36,56 & 1865,44 & 38,27 & 2949,85 & 35,91 & 5871,18 & 38,69 & 11153,27 \\
\hline 6 & $1970-1975$ & 35,16 & 1786,10 & 37,55 & 2669,93 & 36,18 & 5440,73 & 36,15 & 9092,20 \\
\hline 7 & $1975-1980$ & 34,40 & 1737,80 & 37,32 & 2464,37 & 36,67 & 5032,44 & 36,38 & 7630,18 \\
\hline 8 & 1980-1985 & 35,42 & 1744,85 & 37,64 & 2379,84 & 37,57 & 4471,50 & 35,00 & 8745,01 \\
\hline 9 & 1985-1992 & 35,02 & 1674,62 & 37,99 & 2246,41 & 37,63 & 3920,10 & - & - \\
\hline
\end{tabular}

Fuente: Elaboración propia, sobre la base de datos de la Encuesta Nacional de Ocupación y Empleo (ENOE).

Nota: se realizó una prueba de medias para ambos grupos (con ayuda y sin ayuda) y la diferencia en las horas trabajadas resultó estadísticamente significativa.

a El ingreso real se refiere al ingreso mensual en pesos declarado por las personas ocupadas. Para cambiarlo a valores reales se utilizó el Índice Nacional de Precios al Consumidor (INPC) de cada trimestre del período 2005-2010. Se presenta la mediana del salario, ya que debido a la distribución sesgada de los datos es la mejor medida de tendencia central. 
Los hechos estilizados desarrollados en esta sección permiten anticipar lo siguiente: i) el promedio de horas trabajadas por las mujeres que integran las cohortes es mayor cuando hay otra persona adulta en el hogar, y ii) con la presencia de niños menores de 14 años el promedio de horas trabajadas disminuye en cada cohorte. Esto ofrece evidencia empírica de que probablemente la estructura del hogar podría incidir en el tiempo que las mujeres dedican al trabajo remunerado, algo que podría explicarse porque las mujeres también deben elegir la cantidad de horas que destinarán al cuidado de los integrantes del hogar y a la producción de bienes dentro de este en una economía como la mexicana. Además, se constató que el nivel educativo también puede incidir en las horas que las mujeres están dispuestas a destinar al mercado laboral.

\section{Resultados del modelo}

Para determinar el efecto de los factores relacionados con la estructura del hogar en las horas trabajadas por las mujeres se formuló la siguiente especificación econométrica:

Donde:

$$
\operatorname{lnh} h_{i t}=\theta \ln w+X_{i t}+Z_{i t}+u_{i}
$$

lnh es el logaritmo de las horas mensuales trabajadas por las mujeres de la cohorte $i$ en el tiempo $t$. $\ln w$ es el logaritmo del salario real mensual.

$X$ es un vector de variables sociodemográficas y otras características individuales.

$Z$ es un vector de variables asociadas a la estructura del hogar.

$u_{i}$ es el término de error.

No obstante, la estimación de la ecuación podría plantear el problema de endogeneidad en la variable salario real mensual, debido a la posible simultaneidad entre dicha variable y las horas trabajadas. Para hacer frente a este problema se construyeron instrumentos para el salario ${ }^{3}$, cuya especificación econométrica incluye el tipo de cambio real, el nivel de importaciones y el salario mínimo (Robbins, Salinas y Manco, 2009).

En el cuadro 7 se detallan los resultados del modelo de los determinantes de las horas de trabajo de las mujeres a partir de un pseudopanel basado en la muestra representativa de la ENOE a nivel nacional. Los resultados se presentan con cinco tipos diferentes de estimadores para asegurar su robustez estadística. Los datos que figuran en las columnas I a IV se estimaron a partir de un panel que contiene toda la muestra de individuos de la ENOE, desde el tercer trimestre de 2005 al segundo trimestre de 2010, con alrededor de 5,2 millones de observaciones. En la columna (I) se muestra la estimación de la regresión de horas trabajadas sin tomar en cuenta la heterogeneidad entre individuos ni la endogeneidad del salario. En la columna (II) se incluyen efectos cohorte y efectos temporales. Por otra parte, los coeficientes indicados en la columna (III) se calcularon corrigiendo el problema de endogeneidad del salario mediante variables instrumentales. En la columna (IV) se muestran las estimaciones a partir del método de mínimos cuadrados ponderados (WLS). Por último, en la columna (V) se indican las elasticidades de las horas de trabajo de las mujeres a partir del uso de la técnica de pseudopanel dinámico. Es decir, después de obtener la media de las variables de interés para cada cohorte, se obtiene una base de datos temporal y se agregan rezagos de la variable dependiente e instrumentos para controlar la posible endogeneidad del salario real.

Los resultados indican que existe una elasticidad positiva entre el salario y las horas de trabajo de las mujeres, pues ante un aumento del salario estas estarían dispuestas a incrementar el porcentaje de horas que dedican a un trabajo remunerado. Los coeficientes obtenidos en cada uno de los

\footnotetext{
3 Las variables utilizadas para instrumentar el salario fueron: edad, edad al cuadrado, tipo de cambio real, importaciones y salario mínimo.
} 


\section{Cuadro 7}

México: factores determinantes de las horas de trabajo de las mujeres

\begin{tabular}{|c|c|c|c|c|c|}
\hline \multirow[b]{2}{*}{ Variable } & \multicolumn{4}{|c|}{ POOL } & \multirow{2}{*}{$\begin{array}{c}\text { Pseudopane } \\
\text { dinámico HS } \\
\text { (V) }\end{array}$} \\
\hline & (l) & (II) & $\begin{array}{c}\text { Variables } \\
\text { instrumentales } \\
\text { (III) }\end{array}$ & $\begin{array}{l}\text { Mínimos cuadrados } \\
\text { ponderados } \\
\text { (IV) }\end{array}$ & \\
\hline Salario & $\begin{array}{c}0,412 \\
{[0,0005]}\end{array}$ & $\begin{array}{c}0,411 \\
{[0,0005]}\end{array}$ & $\begin{array}{c}0,400 \\
{[0,0108]}\end{array}$ & $\begin{array}{l}0,412 \\
{[0,0005]}\end{array}$ & $\begin{array}{c}0,273 \\
{[0,1333]}\end{array}$ \\
\hline $\begin{array}{l}\text { Presencia de otro adulto } \\
\text { mujer en hogar }\end{array}$ & $\begin{array}{c}0,081 \\
{[0,0009]}\end{array}$ & $\begin{array}{c}0,081 \\
{[0,0009]}\end{array}$ & $\begin{array}{c}0,081 \\
{[0,0009]}\end{array}$ & $\begin{array}{c}0,081 \\
{[0,0009]}\end{array}$ & $\begin{array}{c}0,005 \\
{[0,0030]}\end{array}$ \\
\hline $\begin{array}{l}\text { Presencia de otro adulto } \\
\text { hombre en hogar }\end{array}$ & $\begin{array}{l}-0,003 \\
{[0,0009]}\end{array}$ & $\begin{array}{l}-0,003 \\
{[0,0009]}\end{array}$ & $\begin{array}{l}-0,004 \\
{[0,0010]}\end{array}$ & $\begin{array}{l}-0,003 \\
{[0,0009]}\end{array}$ & $\begin{array}{l}-0,001 \\
{[0,0028]}\end{array}$ \\
\hline Niños de 6 a 14 años & $\begin{array}{l}-0,027 \\
{[0,0005]}\end{array}$ & $\begin{array}{l}-0,027 \\
{[0,0005]}\end{array}$ & $\begin{array}{l}-0,028 \\
{[0,0007]}\end{array}$ & $\begin{array}{l}-0,027 \\
{[0,0005]}\end{array}$ & $\begin{array}{l}0,001 \\
{[0,0037]}\end{array}$ \\
\hline Educación & $\begin{array}{l}-0,032 \\
{[0,0001]}\end{array}$ & $\begin{array}{l}-0,032 \\
{[0,0001]}\end{array}$ & $\begin{array}{l}-0,031 \\
{[0,0010]}\end{array}$ & $\begin{array}{l}-0,032 \\
{[0,0001]}\end{array}$ & $\begin{array}{l}-0,029 \\
{[0,0143]}\end{array}$ \\
\hline Educación-1 & - & - & - & - & $\begin{array}{l}0,010 \\
{[0,0132]}\end{array}$ \\
\hline Educación-2 & - & - & - & - & $\begin{array}{l}-0,020 \\
{[0,0164]}\end{array}$ \\
\hline Edad & $\begin{array}{l}-0,004 \\
{[0,0002]}\end{array}$ & $\begin{array}{l}-0,004 \\
{[0,0001]}\end{array}$ & $\begin{array}{l}-0,004 \\
{[0,0002]}\end{array}$ & $\begin{array}{l}-0,004 \\
{[0,0002]}\end{array}$ & $\begin{array}{c}0,091 \\
{[0,0386]}\end{array}$ \\
\hline Edad $_{-1}$ & - & - & - & - & $\begin{array}{l}-0,187 \\
{[0,0409]}\end{array}$ \\
\hline Constante & $\begin{array}{l}4,121 \\
{[0,0071]} \\
\end{array}$ & $\begin{array}{c}4,093 \\
{[0,0040]} \\
\end{array}$ & $\begin{array}{l}4,139 \\
{[0,0200]} \\
\end{array}$ & $\begin{array}{l}4,122 \\
{[0,0072]} \\
\end{array}$ & $\begin{array}{c}0,0203 \\
{[0,0089]} \\
\end{array}$ \\
\hline Número de observaciones & 1486014 & 1486014 & 1441978 & 1486014 & 261 \\
\hline
\end{tabular}

Fuente: Elaboración propia, sobre la base de datos de la Encuesta Nacional de Ocupación y Empleo (ENOE).

Nota: En todas las regresiones se incluyen regresores ficticios en cada uno de los trimestres. Las regresiones I, II y IV incluyen efectos cohortes y temporales y la II incluye solamente efectos cohortes. []: Desviación estándar.

a Se instrumentó de acuerdo a la metodología planteada previamente.

modelos con diferentes métodos de estimación son consistentes desde el punto de vista de sus valores y significancia estadística. En la columna ( $V$ ), que corresponde a los resultados del pseudopanel dinámico, se observa que ante un aumento del 10\% del salario las mujeres estarían dispuestas a trabajar un 2,7\% más de tiempo. Sin embargo, es importante mencionar que, de acuerdo con los modelos presentados, el salario no es el único factor con un efecto positivo en las horas de trabajo de la mujer en el mercado. Existen otros factores relacionados con la estructura del hogar que también inciden en el tiempo que las mujeres dedican a un trabajo remunerado y que no se han estudiado en profundidad en la literatura previa. Por ejemplo, los resultados del pseudopanel muestran que cuando en el hogar hay otra mujer (mayor de 14 años), que podría constituir una ayuda extra no remunerada en las labores del hogar, las mujeres destinan un mayor tiempo al trabajo remunerado $(0,005)$. Este resultado es consistente con los de las regresiones I, II, III y IV, pues el efecto es el mismo en todas versiones presentadas y todos los casos son estadísticamente significativos. A partir de estos resultados estadísticamente robustos, se puede afirmar que la presencia de otra mujer en el hogar podría suponer una ayuda en las actividades domésticas que normalmente llevan a cabo las mujeres y, en consecuencia, el efecto en las horas que las mujeres destinan al mercado laboral sería positivo (la ayuda extra les permitiría dedicar menos tiempo a las actividades del hogar y al cuidado de sus integrantes). Por lo tanto, las políticas públicas deberían concentrarse en brindar ayuda a las mujeres dentro del hogar para reducir el tiempo que deben dedicar al trabajo doméstico, que muchas veces representa una barrera a su incorporación voluntaria al mercado laboral. En otras palabras, es necesario promover políticas públicas conciliadoras o políticas de reemplazo de algunas tareas no remuneradas, como el servicio de guarderías o ayuda directa para el cuidado de los integrantes 
del hogar que lo requieran (Gammage y Orozco, 2008). No obstante, la presencia en el hogar de al menos un adulto hombre que no tiene un trabajo remunerado (mayor de 14 años) produce un efecto negativo en las horas laborales de las mujeres $(-0,001)$. A pesar de que dicho coeficiente no es estadísticamente significativo (solo en la regresión de pseudopanel), presenta una tendencia robusta en todos modelos reportados.

Por otra parte, de acuerdo con las regresiones I a IV, el efecto en las horas laborales de la presencia de niños de 6 a 14 años de edad es negativo y estadísticamente significativo. Así, cuando en el hogar hay niños, las mujeres reducen el tiempo que dedican al trabajo remunerado. Esto puede explicarse por la demanda de tiempo que el cuidado de los niños representa para las mujeres (Pedrero, 2009). De lo anterior se infiere que una reducción de los costos del cuidado de los niños aumenta las horas de trabajo de las mujeres en el mercado laboral (Attanasio, Low y Sánchez, 2008). Otro hallazgo relevante y consistente en todas las estimaciones se refiere al impacto negativo del nivel educativo en las horas que las mujeres destinan al trabajo remunerado. Para ello, se consideró la variable que indica los años de escolaridad de las mujeres y, debido a que con niveles más altos de escolaridad se obtienen ingresos más elevados y mejores condiciones de empleo (Domínguez y Brown, 2013), es posible que en este caso el efecto sustitución tenga mayor peso. En otras palabras, al tener un nivel de ingresos considerable, las mujeres optan por reducir las horas de trabajo en el mercado laboral y destinarlas a otras actividades. Otro factor que incide negativamente en las horas que las mujeres destinan a un trabajo remunerado es la edad, pues dicho resultado también es robusto en todas las versiones presentadas en el cuadro 7. A partir de esto se puede afirmar que cuantos más años tengan las mujeres, menos dispuestas estarán a destinar horas a un trabajo remunerado.

En general, las variables que inciden positivamente en las horas laborales de las mujeres y cuyos resultados presentan una apropiada robustez estadística son: i) el salario, y ii) la presencia de otra mujer mayor de 14 años en el hogar. Por otra parte, los factores que inciden negativamente en las horas de trabajo de las mujeres y tienen consistencia en todas las regresiones son: i) la presencia de un hombre mayor de 14 años; ii) la presencia de niños que tienen entre 6 y 14 años de edad; iii) el nivel educativo, y iv) la edad. Sobre esta base se puede afirmar que la estructura del hogar es un elemento relevante en la determinación de las horas que las mujeres destinan al trabajo remunerado y que esto podría relacionarse con el tiempo que estas destinan a ese tipo de actividades.

\section{Comentarios finales}

El objetivo de este estudio ha sido determinar si la estructura del hogar influye de alguna manera en las horas que las mujeres destinan al trabajo remunerado en el mercado laboral. Los resultados obtenidos con la técnica de pseudopanel sugieren que las horas de trabajo de una mujer sí dependen del número de adultos y de niños que viven en el hogar. Específicamente, se encontró evidencia de que la presencia de niños menores de edad afecta negativamente la disponibilidad de tiempo de las mujeres. Esto se podría relacionar directamente con el cuidado y la atención que estos integrantes requieren de la mujer en función de su papel tradicional dentro del hogar, ocupando así la mayor parte de su tiempo y reduciendo simultáneamente su disponibilidad de horas para trabajar. En este contexto, es posible afirmar que las mujeres realizan en el hogar una cantidad considerable de trabajo no remunerado que limita sus posibilidades de incorporarse al mercado laboral. Los resultados del presente estudio permiten ofrecer algunas sugerencias de política pública que se enfoquen en la reducción del tiempo que las mujeres dedican a las actividades domésticas y el cuidado del hogar. Una primera recomendación consiste en diseñar y desarrollar acciones encaminadas a generar e incentivar la ayuda dentro de los hogares. Si la presencia de otra mujer permite reducir la carga de trabajo en el hogar y promueve un aumento de las horas de trabajo femenino, se requieren políticas públicas destinadas a reemplazar dicha ayuda, para evitar una transmisión de trabajo no pagado de 
una mujer a otra. Por lo tanto, se necesitan políticas públicas - como la ampliación del número de guarderías públicas y el desarrollo de programas escolares - que den mayor flexibilidad de horario a las mujeres. Es importante que se desarrollen políticas públicas dirigidas al apoyo de las actividades domésticas, sobre todo aquellas relacionadas con los integrantes del hogar y su cuidado. Con este tipo de acciones las horas de trabajo aumentarían, pues las mujeres estarían en mejores condiciones para ofrecer un mayor número de horas al mercado de trabajo. A su vez, esto les permitiría mejorar su calidad de vida, si ellas así lo deciden de manera voluntaria. Las actividades domésticas, como el cuidado de la familia y la producción de bienes dentro del hogar, constituyen uno de los principales elementos que limitan la disponibilidad de tiempo de las mujeres para trabajar formalmente, un aspecto menos relevante en el caso de los hombres (INEGI, 2012).

\section{Bibliografía}

Acosta, E., M. Perticará y C. Ramos (2006), Empleo femenino: oferta laboral y cuidado infantil, Washington, D.C., Banco Interamericano de Desarrollo.

Arceo-Gómez, E. y R. Campos-Vázquez (2010), "Labor supply of married women in Mexico: 1990-2000", Documento de Trabajo, № 16, El Colegio de México.

Attanasio, O., H. Low y M. Sánchez (2008), "Explaining changes in female labor supply in a life-cycle model", American Economic Review, vol. 98, № 4, Nashville, Tennessee, American Economic Association.

Bassi, M. (2003), "Do really matter? Understanding female force participation", Octava Reunión de la Asociación de Economía de América Latina y el Caribe, Ciudad de México.

Dasgupta, P. y B. Goldar (2005), "Female labour supply in rural India: an econometric analysis", Institute of Economic Growth.

Deaton, A. (1997), The Analysis of Household Surveys: a Microeconometric Approach to Development Policy, Baltimore, Johns Hopkins University Press.

(1985), "Panel data from a time series of cross-sections", Journal of Econometrics, vol. 30, № 1-2, Amsterdam, Elsevier.

Dessing, M. (2002), "Labor supply, the family and poverty: the S-shaped labor supply curve", Journal of Economic Behavior and Organization, vol. 49, № 4, Amsterdam, Elsevier.

Domínguez, L. y F. Brown (2013), "Diferencias de género en la elección del sitio de trabajo en un contexto de crisis", Revista CEPAL, № 111 (LC/G.2597-P), Santiago, Comisión Económica para América Latina y el Caribe (CEPAL).

Gammage, S. y M. Orozco (2008). "El trabajo productivo no remunerado dentro del hogar: Guatemala y México", serie Estudios y Perspectivas, № 103 (LC/L.2983-P), Ciudad de México, Sede Subregional de la CEPAL en México.

INEGI (Instituto Nacional de Estadística y Geografía) (s/f), "Encuesta Nacional de Ocupación y Empleo (ENOE) 2005-2010", Ciudad de México.

_ (2012), Encuesta Nacional sobre el Uso del Tiempo (ENUT) 2009 [en línea] http://internet.contenidos.inegi. org.mx/contenidos/productos//prod_serv/contenidos/espanol/bvinegi/productos/encuestas/especiales/ enut/2009/ENUT_2009_MTB.pdf.

Juhn, C. y K.M. Murphy (1996), "Wage inequality and family labor supply", NBER Working Paper Series, $N^{\circ}$ 5459, Cambridge, Massachusetts, National Bureau Of Economic Research.

Licona, G. (2000), "Reshaping the labor supply curve for the poor", documento presentado en la Reunión de la Asociación de Economía de América Latina y el Caribe, Río de Janeiro.

Ludin, D., E. Mörk y B. Öckert (2008), "How far can reduced childcare prices female labor supply?", Labour Economics, vol. 15, № 4, Amsterdam, Elsevier.

Pedrero, M. (2009), Valor económico del trabajo doméstico en México. Aportaciones de mujeres y hombres, Ciudad de México, Instituto Nacional de las Mujeres (INMUJERES).

Robbins, D., D. Salinas y A. Manco (2009), "La oferta laboral femenina y sus determinantes: evidencia para Colombia con estimativas de cohortes sintéticas", Lecturas de Economía, № 70.

Schultz, P. (1990), "Testing the neoclassical model of family labor supply and fertility", Journal of Human Resources, vol. 25, N 4, Wisconsin, University of Wisconsin Press.

Warunsiri, S. y R. McNown (2010), "The returns to education in Thailand: a pseudo-panel approach", World Development, vol. 38, № 11, Amsterdam, Elsevier. 